\title{
One-dimensional unsteady solute transport along unsteady flow through inhomogeneous medium
}

\author{
Sanjay K Yadav ${ }^{1}$, Atul KumaR ${ }^{2}$, Dilip K Jaiswal ${ }^{2}$ and Naveen Kumar ${ }^{1, *}$ \\ ${ }^{1}$ Department of Mathematics, Banaras Hindu University, Varanasi 221 005, India. \\ ${ }^{2}$ Department of Mathematics, Lucknow University, Lucknow 226 007, India. \\ *e-mail:nks_1953@yahoo.co.in
}

\begin{abstract}
The one-dimensional linear advection-diffusion equation is solved analytically by using the Laplace integral transform. The solute transport as well as the flow field is considered to be unsteady, both of independent patterns. The solute dispersion occurs through an inhomogeneous semi-infinite medium. Hence, velocity is considered to be an increasing function of the space variable, linearly interpolated in a finite domain in which solute dispersion behaviour is studied. Dispersion is considered to be proportional to the square of the spatial linear function. Thus, the coefficients of the advection-diffusion equation are functions of both the independent variables, but the expression for each coefficient is considered in degenerate form. These coefficients are reduced into constant coefficients with the help of a new space variable, introduced in our earlier works, and new time variables. The source of the solute is considered to be a stationary uniform point source of pulse type.
\end{abstract}

\section{Introduction}

Pollution can be classified in many ways. On the basis of the environment where it occurs most, it can be classified as air pollution, soil pollution, surface water pollution and groundwater pollution. Its source may be natural or anthropogenic. One type of source of these pollutions is a point source. Stationary point sources include volcanoes, factories, electric power plants, mineral smelters, petroleum refineries and different small-scale industries; while mobile point sources include all sorts of transport vehicles on road, rail or air. A point source is a specific site in a medium (air, water or soil) where the discharge of pollutant's solute particles, in the form of effluents or particulate matters from such a source, enter the environment and are transported away from the source due to diffusion and advec- tion. Groundwater pollution occurs due to infiltration of wastes through rainwater, from garbage disposal sites, septic tanks and mines, and discharge from surface water bodies polluted due to industrial and municipal influents. Much of the natural impurities in rainwater and wastes get removed during infiltrating through soil strata but overexploitation of groundwater increases pollution.

The pollutant's solute transport from a source along the flow field through a medium of air or water is described by a partial differential equation of parabolic type derived on the principle of conservation of mass, and is known as the advectiondiffusion equation (ADE). Also, ADE has similar role in life processes (Shukla et al 2008). Its analytical solutions are of fundamental importance in understanding and describing physical phenomena because they explicitly take into account all

Keywords. Advection; diffusion; inhomogeneous medium; degenerate form; variable coefficients. 
parameters of the problem. The literature presents several methods to analytically solve ADE. The literature also features the use of mathematical substitutions in obtaining analytical solutions to partial differential equations. Such methods are very well reviewed in a recent paper (Guerrero et al 2009) in which a formal exact solution of the linear advection-diffusion transport equation with constant coefficients for both transient and steady state regimes is presented by using a classical substitution. At this point it is necessary to mention the existence of many closed-form solutions either in integral form or series formulations, with the main property that both solutions are equivalent. Among them we mention some works (Rounds 1955; Smith 1957; Ogata 1970; Marino 1974; Scriven and Fisher 1975; Demuth 1978). All of these studies were done in one-dimension, and have been compiled (van Genuchten and Alves 1982; Lindstrom and Boersma 1989). In earlier studies, a comparable substitution was used to obtain a transient, one-dimensional solution for miscible fluid displacement in finite length beds (Brenner 1962). An analytical solution for reactive solutes with linear adsorption, a source/sink term, a finite domain, and continuous-flux plugtype inlet conditions at the inlet boundary was presented (Selim and Mansell 1976). Later, an equivalent substitution was used by Golz (2003) for studying convective-dispersive solute transport with constant production, first-order decay and equilibrium sorption in a porous medium. An analytical technique for solving ADE with hyperbolic asymptotic distance-dependent dispersivity was presented (Chen et al 2008), in which the solution was derived by applying the extended power series method coupled with the Laplace transform. An analytical model was presented for solute transport in rivers, including transient storage in hyporheic zones (Smedt 2007), which consists of an ADE for transport in the main channel with a sink term describing diffusive solute transfer to the hyporheic zone. A classification of existing modelling approaches was proposed and theoretical models are reviewed with an emphasis on their mathematical formulations and their capacities to model the scale effect in longitudinal dispersion (Frippiat and Holeyman 2008).

Analytical solutions of advective-diffusive transport problems continue to be of interest in validating more comprehensive numerical solutions of the governing transport equations. Until recently it was frequently the case that numerical schemes were only tested against known analytical solutions to the ADE with constant coefficients. A reason for this was that there were very few analytical solutions to the ADE with variable coefficients, with few notable exceptions (Shevelev 1969;
Zoppou and Knight 1999). The limitation of such solutions was discussed by Neelz (2006). In the last few years, special attention has been given to searching for analytical solutions. Seven different classes of linear boundary value problems of heat and mass diffusion were studied (Mikhailov and Ozisik 1984), and resulted in the development of three-dimensional general solutions for each of the seven classes of problems by using the generalized integral transform technique (GITT) in the context of separation-of-variable or eigen function expansions. This technique is much used in recent works (Cotta and Mikhailov 1997; Wortmann et al 2005; Cassol et al 2009; Moreira et al 2009). Advectiondiffusion multilayer method (Costa et al 2006) is another method for use in this context.

The Laplace integral transform technique (LITT) is simpler and more viable for solving an initial and boundary value dispersion problem than any other method. Using LITT, we obtained analytical solutions of some dispersion problems in our recent works (Jaiswal et al 2009; Kumar et al 2009, 2010), in which the two coefficients, one of which represents dispersion parameter and the other represents velocity of the flow field, were considered as either temporally dependent or spatially dependent. In the present study, both the coefficients of one-dimensional ADE are considered in more general form - each coefficient is a function of both the independent variables (space and time variables) expressed in degenerate form. The reason and usefulness of choosing such variable coefficients is explained in the following section. It brings the assumptions of the present study closer to real problems. In the present study, the temporal dependence of dispersion or advection is considered to be either exponentially decreasing or increasing in nature. Other time-dependent functions may also be considered if an explicit relationship between the two new time variables, such as the one obtained in the present study, is possible. In most of the real-world unsteady solute transport phenomena, dispersion as well as convection is exponential in nature. Therefore, the analytical solution proposed in this study is expected to be more useful in validating numerical solutions of a more general nature. The analytical solution is obtained using LITT, which describes the unsteady (of exponential nature) solute transport from a pulse-type stationary point source of uniform nature along the unsteady flow (also of exponential nature) through a semi-infinite medium of linearly increasing inhomogeneity. From this solution, those obtained in our earlier works cited above, and in the dispersion problems with constant coefficients, may be obtained as particular cases, as explained in section 3.1. These solutions are compared with each other by plotting the 
concentration values. This validates the integrity of the solution obtained in this study.

\section{Unsteady solute transport along unsteady flow through inhomogeneous medium}

The linear advection-diffusion partial differential equation in one dimension may be written as:

$$
\frac{\partial c}{\partial t}=\frac{\partial}{\partial x}\left(D(x, t) \frac{\partial c}{\partial x}-u(x, t) c\right),
$$

where $c$ is the solute concentration at a position $x$ at time $t$, the first coefficient $D(x, t)$ is the solute dispersion parameter and the second coefficient $u(x, t)$ is the velocity of the medium transporting the solute particles. In the present study, this equation is solved for a dispersion problem in which both the coefficients remain variable, i.e., they remain functions of independent variables $x$ and $t$. The medium is considered to be of semi-infinite extent and is inhomogeneous.

Owing to the inhomogeneous nature of the medium, the velocity of the flow field transporting the solute particles along its downstream is considered spatially dependent. An increasing function for it is linearly interpolated in terms of a position variable in a finite longitudinal region, in which concentration values are evaluated (Jaiswal et al 2009; Kumar et al 2009, 2010). Further, the velocity is also considered temporally dependent. The expression for velocity is written in degenerate form as:

$$
u(x, t)=u_{0} f_{1}\left(m_{1} t\right)(1+a x),
$$

where $a$ is the inhomogeneity parameter whose dimension is the inverse of that of the space variable. Its different values represent media of varying inhomogeneity. The variable $m_{1}$ is an unsteady flow parameter whose dimension is the inverse of that of $t$. The solute dispersion is also considered unsteady but not in the same proportion in which velocity varies with time. Its spatial dependence (due to the inhomogeneity of the medium) is considered to vary with the square of the spatial dependency of the velocity (Scheidegger 1957), and so we consider the following equation:

$$
D(x, t)=D_{0} f_{2}\left(m_{2} t\right)(1+a x)^{2},
$$

where $m_{2}$ is the another unsteady dispersion parameter for solute transport whose dimension is the inverse of that of the time variable. While choosing expressions for $f_{1}\left(m_{1} t\right)$ and $f_{2}\left(m_{2} t\right)$, it is ensured that $f_{1}\left(m_{1} t\right)=1$ for $m_{1}=0$ and $f_{2}\left(m_{2} t\right)=1$ for $m_{2}=0$. Both represent steady flow and steady solute transport, respectively. Also, it is ensured that $f_{1}\left(m_{1} t\right)=f_{2}\left(m_{2} t\right)=1$ at $t=0$. This represents the velocity and the dispersion at the initial stage. The temporally dependent and spatially dependent expressions are in non-dimensional terms, and so the constants $u_{0}$ and $D_{0}$ in equations (2) and (3) may be referred to as uniform velocity of dimension $\left(L T^{-1}\right)$ and the initial dispersion coefficient of dimension $\left(L^{2} T^{-1}\right)$, respectively. Thus, the advection-diffusion equation (1) may be written as:

$$
\begin{aligned}
\frac{\partial c}{\partial t}= & \frac{\partial}{\partial x}\left(D_{0} f_{2}\left(m_{2} t\right)(1+a x)^{2} \frac{\partial c}{\partial x}\right. \\
& \left.-u_{0} f_{1}\left(m_{1} t\right)(1+a x) c\right) .
\end{aligned}
$$

\section{Initial and boundary value problem: analytical solution}

The medium through which solute dispersion occurs is considered to be of semi-infinite extent along the longitudinal direction. Initially it is considered solute free. The source of the pollutant's solute is considered to be a point source and to be situated at the origin of the medium. It is of pulse type and uniform nature. In other words, the input concentration at the origin remains uniform up to a certain time period until the source is present, and just after the source is eliminated, it becomes zero. For example, smoke coming out of a chimney of a factory or waste meeting a riverbed at a uniform rate vanishes as soon as the factory or the drainage system is closed.

A flux-type homogeneous condition is assumed at the far end of the medium. Thus, the analytical solution, $c(x, t)$ of the advection-diffusion equation (4) is desired with respect to the following initial and boundary conditions:

$$
\begin{gathered}
c(x, t=0)=0, \quad x \geq 0, \\
c(x=0, t)= \begin{cases}C_{0} ; & 0<t \leq t_{0} \\
0 ; & t>t_{0}\end{cases} \\
\frac{\partial c}{\partial x}=0 ; \quad x \rightarrow \infty, \quad t \geq 0 .
\end{gathered}
$$

The basic approach is to reduce the variable coefficients of the advection-diffusion equation (4) into constant coefficients. This is initiated with the introduction a new space variable through a transformation (Jaiswal et al 2009; Kumar et al 2010):

$$
X=\frac{1}{a} \log (1+a x),
$$


and a new time variable through another transformation (Crank 1975)

$$
T^{*}=\int_{0}^{t} f_{1}\left(m_{1} t\right) d t .
$$

Advection-diffusion equation (4) becomes:

$$
\begin{aligned}
\frac{\partial c}{\partial T^{*}}= & D_{0} \frac{f_{2}\left(m_{2} t\right)}{f_{1}\left(m_{1} t\right)} \frac{\partial^{2} c}{\partial X^{2}} \\
& -u_{0}\left\{1-a \frac{D_{0} f_{2}\left(m_{2} t\right)}{u_{0} f_{1}\left(m_{1} t\right)}\right\} \frac{\partial c}{\partial X}-a u_{0} c .
\end{aligned}
$$

Further, the first-order decay term in the above partial differential equation is eliminated through another transformation:

$$
c=C \exp \left(-a u_{0} T^{*}\right) .
$$

As a result, the initial and boundary value problem becomes:

$$
\begin{gathered}
\frac{\partial C}{\partial T^{*}}=D_{0} \frac{f_{2}\left(m_{2} t\right)}{f_{1}\left(m_{1} t\right)} \frac{\partial^{2} C}{\partial X^{2}} \\
-u_{0}\left\{1-a \frac{D_{0} f_{2}\left(m_{2} t\right)}{u_{0} f_{1}\left(m_{1} t\right)}\right\} \frac{\partial C}{\partial X}, \\
C\left(X, T^{*}=0\right)=0, \quad X \geq 0, \\
C\left(X=0, T^{*}\right)= \begin{cases}C_{0} \exp \left(a u_{0} T^{*}\right) ; & 0<T^{*} \leq T_{0}^{*}, \\
0 ; & T^{*}>T_{0}^{*}\end{cases}
\end{gathered}
$$

and

$$
\frac{\partial C}{\partial X}=0 ; \quad X \rightarrow \infty, \quad T^{*} \geq 0 .
$$

Let

$$
f_{3}(m t)=1-a \frac{D_{0} f_{2}\left(m_{2} t\right)}{u_{0} f_{1}\left(m_{1} t\right)}=1-\lambda \frac{f_{2}\left(m_{2} t\right)}{f_{1}\left(m_{1} t\right)}
$$

be defined as another time-dependent expression in non-dimensional variable (term), $m t$, where $\lambda=$ $\left(a D_{0} / u_{0}\right)$, is a non-dimensional parameter. So, equation (12) may be written as:

$$
\begin{aligned}
\frac{1}{f_{3}(m t)} \frac{\partial C}{\partial T^{*}}= & D_{0} \frac{f_{2}\left(m_{2} t\right)}{f_{1}\left(m_{1} t\right) f_{3}(m t)} \\
& \times \frac{\partial^{2} C}{\partial X^{2}}-u_{0} \frac{\partial C}{\partial X} .
\end{aligned}
$$

Further, the introduction of another non-dimensional space variable through a transformation

$$
Z=X \frac{f_{1}\left(m_{1} t\right)}{f_{2}\left(m_{2} t\right)} f_{3}(m t)
$$

and lastly the introduction of another time variable through a separate transformation

$$
T=\int_{0}^{t} \frac{f_{1}^{2}\left(m_{1} t\right)}{f_{2}\left(m_{2} t\right)} f_{3}^{2}(m t) d t,
$$

accomplishes the desired objective. The initial and boundary value problem with constant coefficients in $(Z, T)$ domain is obtained as follows:

$$
\begin{gathered}
\frac{\partial C}{\partial T}=D_{0} \frac{\partial^{2} C}{\partial Z^{2}}-u_{0} \frac{\partial C}{\partial Z} \\
0 \leq Z<\infty, \quad T>0, \\
C(Z, T=0)=0, \quad Z \geq 0 \\
C(Z=0, T)= \begin{cases}C_{0} \exp \left(\gamma a u_{0} T\right) ; & 0<T \leq T_{0} \\
0 ; & T>T_{0}\end{cases} \\
\text { where } \gamma=(1-\lambda)^{-2}, \text { and } \\
\frac{\partial C}{\partial Z}=0 ; \quad Z \rightarrow \infty, \quad T \geq 0
\end{gathered}
$$

It may be noted here too that for expressions of $f_{1}\left(m_{1} t\right)$ and $f_{2}\left(m_{2} t\right)$, the dimension of $T$ will be that of $t$; hence, it is referred to as new time variable. Additionally, these expressions are so chosen that for $t=0, T=0$ is ensured so that the nature of initial condition does not change.

Further, to write the input condition (22), the time variable $T^{*}$ occurring in condition (14) has to be expressed in terms of another time variable, $T$. For this, we chose two independent temporally dependent expressions: the one of exponentially increasing nature and the other of exponentially decreasing nature, for same unsteadiness parameter $\left(m_{1}=m_{2}=m\right)$. So, we consider

$$
f_{1}(m t)=\exp (m t)
$$

and

$$
f_{2}(m t)=\exp (-m t) .
$$

From transformation (9), we have:

$$
T^{*}=\int_{0}^{t} \exp (m t) \mathrm{d} t=\frac{1}{m}[\exp (m t)-1]
$$

or

$$
\exp (m t)=1+m T^{*}
$$

or

$$
\exp (-m t)=\left(1+m T^{*}\right)^{-1}
$$


Also, from transformation (19), we have:

$$
T=\int_{0}^{t} \exp (3 m t)\{1-\lambda \exp (-2 m t)\}^{2} \mathrm{~d} t
$$

or

$$
\begin{aligned}
T= & \frac{1}{3 m} \exp (3 m t)-\frac{\lambda^{2}}{m} \exp (-m t) \\
& -\frac{2 \lambda}{m} \exp (m t)-\frac{1}{3 m}+\frac{\lambda^{2}}{m}+\frac{2 \lambda}{m}
\end{aligned}
$$

or

$$
\begin{aligned}
T= & \frac{1}{3 m}\left(1+m T^{*}\right)^{3}-\frac{\lambda^{2}}{m}\left(1+m T^{*}\right)^{-1} \\
& -\frac{2 \lambda}{m}\left(1+m T^{*}\right)-\frac{1}{3 m}+\frac{\lambda^{2}}{m}+\frac{2 \lambda}{m} .
\end{aligned}
$$

The unsteady parameter $m$ is considered to be much smaller than 1, and so its second- and higherdegree terms in the binomial expansions in the above equation are omitted. So, we obtain

$$
T=(1-\lambda)^{2} T^{*} \quad \text { or } \quad T^{*}=\gamma T,
$$

where $\gamma$ is defined in condition (22).

Now, the analytical solution of the initial and boundary value problem defined by equations (20)(23) may be obtained by using LITT, as follows:

$$
c(x, t)= \begin{cases}F(x, t) ; & 0<t \leq t_{0}, \\ F(x, t)-F\left(x, t-t_{0}\right) ; & t>t_{0}\end{cases}
$$

where

$$
\begin{gathered}
F(x, t)=\frac{C_{0}}{2}\left[\exp \left\{\left(\frac{u_{0}}{2 D_{0}}-\frac{\mu}{\sqrt{D_{0}}}\right) Z\right\}\right. \\
\times \operatorname{erfc}\left\{\frac{Z}{2 \sqrt{D_{0} T^{*} / \gamma}}-\mu \sqrt{T^{*} / \gamma}\right\} \\
+\exp \left\{\left(\frac{u_{0}}{2 D_{0}}+\frac{\mu}{\sqrt{D_{0}}}\right) Z\right\} \\
\left.\times \operatorname{erfc}\left\{\frac{Z}{2 \sqrt{D_{0} T^{*} / \gamma}}+\mu \sqrt{T^{*} / \gamma}\right\}\right], \\
Z=\frac{f_{1}\left(m_{1} t\right) f_{3}(m t)}{a f_{2}\left(m_{2} t\right)} \log (1+a x), \\
f_{1}\left(m_{1} t\right)=\exp (m t) \quad \text { for } m_{1}=m,
\end{gathered}
$$

$$
\begin{gathered}
f_{2}\left(m_{2} t\right)=\exp (-m t) \text { for } m_{2}=m, \\
f_{3}(m t)=1-\lambda \exp (-2 m t), \\
\lambda=\frac{a D_{0}}{u_{0}}, \quad T^{*}=\frac{1}{m}[\exp (m t)-1] \\
\mu=\sqrt{\frac{u_{0}^{2}}{4 D_{0}}+\gamma a u_{0}},
\end{gathered}
$$

and

$$
\gamma=\frac{1}{(1-\lambda)^{2}}
$$

Relationship (26) may also be obtained for the same two expressions in equation (24) but in reversed role, that is, for the following combination of the two time-dependent functions:

$$
f_{1}(m t)=\exp (-m t)
$$

and

$$
f_{2}(m t)=\exp (m t) .
$$

So, solution (28) may be used for the above combination of the two functions, but in that case,

$$
f_{3}(m t)=1-\lambda \exp (2 m t)
$$

and

$$
T^{*}=\frac{1}{m}[1-\exp (-m t)] .
$$

It may further be noted that the solution in the case of uniform continuous input concentration may be written as:

$$
c(x, t)=F(x, t) ; \quad t>0,
$$

where $F(x, t)$ will be given by equation $(28)$.

\subsection{Particular solutions}

The key feature of this solution lies in obtaining the solutions in all possible cases dealt with the different theories of solute dispersion, which are discussed below:

- $m_{1}=m_{2}=0$. It represents steady solute transport along steady flow but through inhomogeneous medium, the inhomogeneity being defined as in the above section. We have $f_{1}\left(m_{1} t\right)=1$, $f_{2}\left(m_{2} t\right)=1, f_{3}(m t)=1-\lambda, T^{*}=t, Z=(1-\lambda) /$ $a \log (1+a x)$, and so on. Solution (27) reduces to the solution given by equation (50) (Jaiswal et al 2009) and the solution (30) reduces to the solution given by equation (18) (Kumar et al 2010). 
- $m_{1}=0 ; m_{2}=m$. This case represents the unsteady solute transport along steady flow through the inhomogenenous porous media (Matheron and de Marsily 1980), and the corresponding analytical solution may be obtained from solution (27) for pulse-type uniform input or from the solution (30) for continuous uniform input, with proper substitution in the expression (28) of $F(x, t)$. The similar problem but through a homogeneous medium is dealt with in section 2.1 (Jaiswal et al 2009).

- $m_{1}=m ; m_{2}=0$. This case represents the steady solute transport along unsteady flow through the inhomogenenous porous media, and the corresponding analytical solution may be obtained with the proper substitution.

- $f_{2}(m t)$ is proportional to $f_{1}(m t)$. In this case, solute dispersion becomes proportional to the velocity (Rumer 1962).

- $f_{2}(m t)$ is proportional to square of $f_{1}(m t)$. In this case, solute dispersion becomes proportional to the square of the velocity (Scheidegger 1957).

\section{Illustrations and discussions}

To illustrate solution (27), the input values are chosen as: reference concentration, $C_{0}=1.0$; initial velocity, $u_{0}=0.60(\mathrm{~km} /$ year $)$; initial dispersion coefficient, $D_{0}=0.71\left(\mathrm{~km}^{2} /\right.$ year $)$; inhomogeneous parameter, $a=0.1(\mathrm{~km})^{-1}$; and unsteady param-

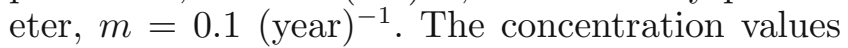
are evaluated in a finite longitudinal space domain, $0 \leq x(\mathrm{~km}) \leq 1.0$, of the semi-infinite medium. So, due to inhomogeneity of the medium only, i.e., at $t=0$, from equation (2), it may be seen that the velocity varies from a value 0.60 at the origin $(x=0)$ to a value 0.66 at the end $(x=1)$ of the finite space domain considered. Figure 1(a) is drawn in the time domain $t<t_{0}$, at $t$ (year) $=0.1,0.7,1.3,1.9$, and figure $1(\mathrm{~b})$ is drawn in the domain $t>t_{0}$ at $t($ year $)=2.2,2.8,3.4,4.0$. The time of elimination of the source of pollution is considered as $t_{0}=2.0$ (year). Thus, the time interval is considered to be 0.6 years. The full line curves (unmarked) in both the figures are drawn for the functions defined by equation (24), i.e., the unsteady pattern of the flow field is considered to be of exponentially increasing nature, while that of the solute transport is of exponentially decreasing nature. It may be observed that in the presence of a uniform pollutant source, concentration values decrease with position and increase with time. In almost 2 years, the longitudinal domain becomes polluted at the level slightly below that of the input concentration. Once the source of the pollution is removed, the input concentration becomes zero and, at a particular position concentration level, starts decreasing
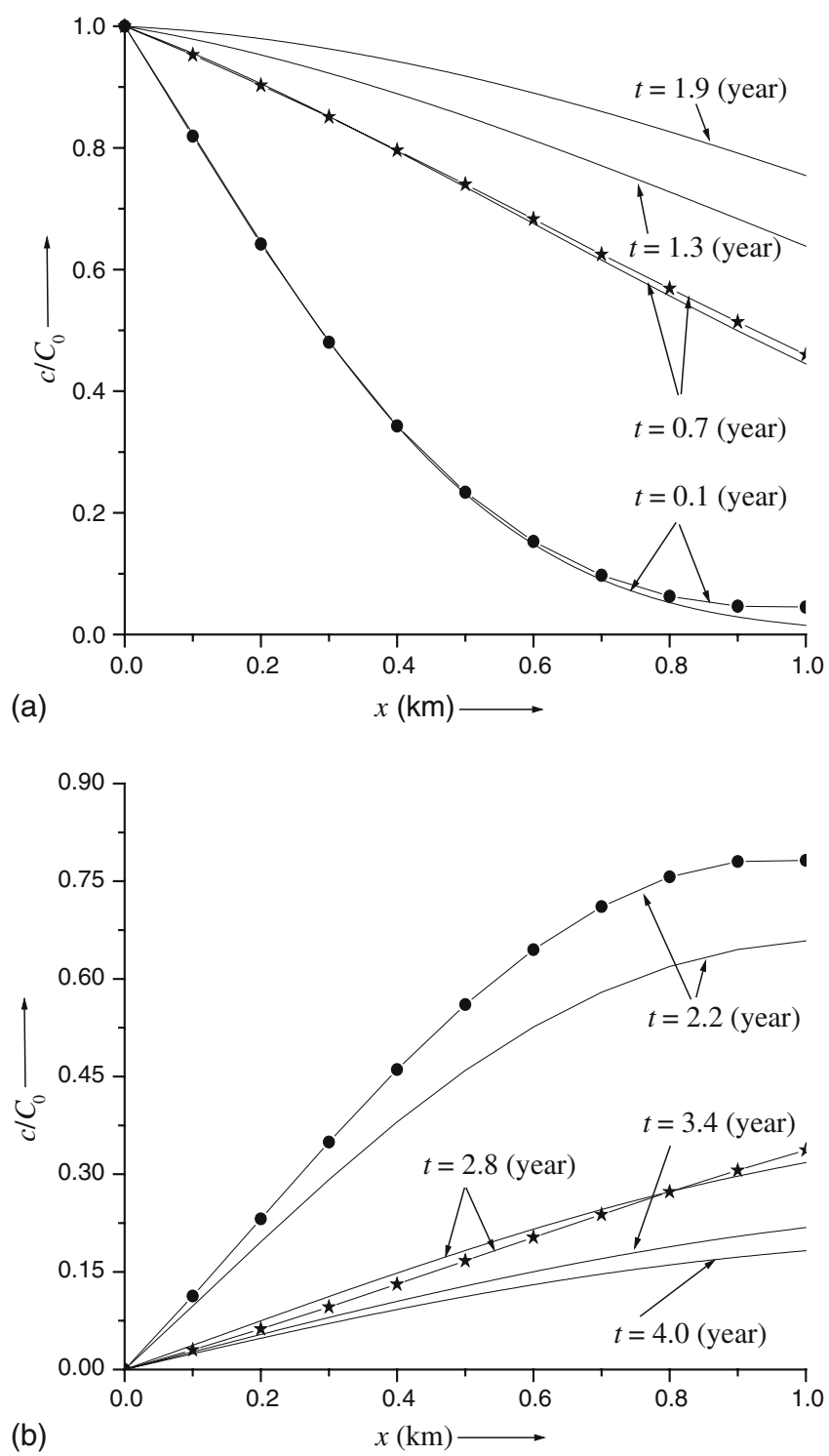

Figure 1. Solute transport due to exponentially decreasing time-dependent dispersion along exponentially increasing time-dependent flow through inhomogeneous medium (a) in the presence of the uniform pulse-type point source, shown by the four unmarked curves. The curve marked with circles shows the numerical solution of the same problem. The curve marked with stars shows the analytical solution of the ADE with constant coefficients subjected to same conditions and (b) in the absence of the uniform pulse-type point source, shown by the four unmarked curves. The curve marked with circles shows the numerical solution of the same problem. The curve marked with stars shows the analytical solution of the ADE with constant coefficients subjected to same conditions.

with time and appears to settle at a minimum level after 2 years. The increase in concentration level (in the presence of the source) or decrease in the same (in the absence of the source) is faster at the initial stage.

To establish the accuracy and integrity of the solution obtained in the present study, it is compared 
with two more solutions. One is the numerical solution of the same problem and the other one is the analytical solution of the problem with constant coefficients (van Genuchten and Alves 1982, problem $\mathrm{A} 1$ for the value of retardation factor, $R=1$ ), which is that of the ADE

$$
\frac{\partial c}{\partial t}=D_{0} \frac{\partial^{2} c}{\partial x^{2}}-u_{0} \frac{\partial c}{\partial x}
$$

along with initial and boundary conditions given by equations (5)-(7). The former solution is obtained by using the two-level explicit finite difference method. The step sizes along the two axes of independent variables are chosen according to the stability norm. This solution is shown by a full line curve marked with circles at $t=0.1$ (year) and $t=2.2$ (year) in figure $1(\mathrm{a})$ and $1(\mathrm{~b})$, respectively. The latter solution is shown by the curve marked by stars at $t=0.7$ (year) and $t=2.8$ (year) in both the figures respectively. The difference between the concentration values in the latter comparison will become more significant in case higher values of unsteady parameter $m$ and inhomogeneity parameter $a$ are chosen. Both the comparisons validate the result of the present study.

In view of the particular cases discussed in section 3.1, the concentration values are evaluated from solution (27) and are plotted in figure 2(a and b) for the following five cases:

- exponentially decreasing time-dependent dispersion along exponentially increasing time-dependent flow, the forms given by equation (24),

- exponentially increasing time-dependent dispersion along exponentially decreasing time-dependent flow, the forms given by equation (29),

- steady dispersion along steady flow, discussed as the first particular case in section 3.1,

- exponentially decreasing time-dependent dispersion along steady flow, discussed as the second particular case in section 3.1, and

- steady dispersion along exponentially decreasing time-dependent flow, discussed as the third particular case in section 3.1.

In figure 2(a), all the five concentration curves are depicted at $t=1.3$ (year), but at $t=0.1$ (year), only the curves for the first two cases are drawn. Similarly, in figure 2(b), the five curves are drawn at $t=3.4$ (year) and at $t=2.2$ (year) only two curves are drawn. It may be observed that in the presence of the source of pollution, solute transport is the slowest in the first case, is the fastest in the last case, and that of the third case lies in between the second and the fourth cases, the solute transport in the latter being the fastest among the three cases. Similarly, in the absence of the source, the rehabilitation is the fastest in the first case.

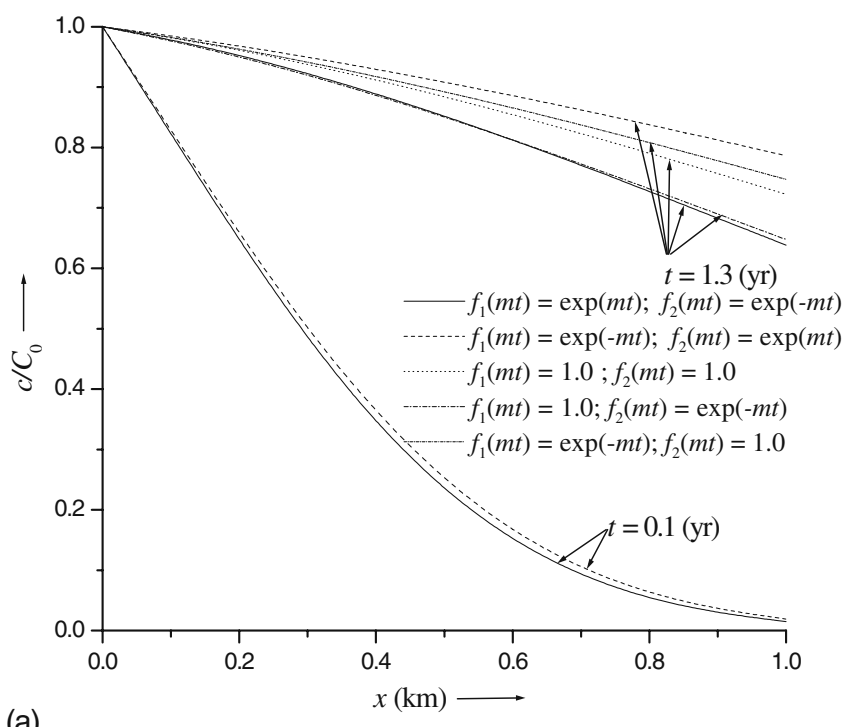

(a)

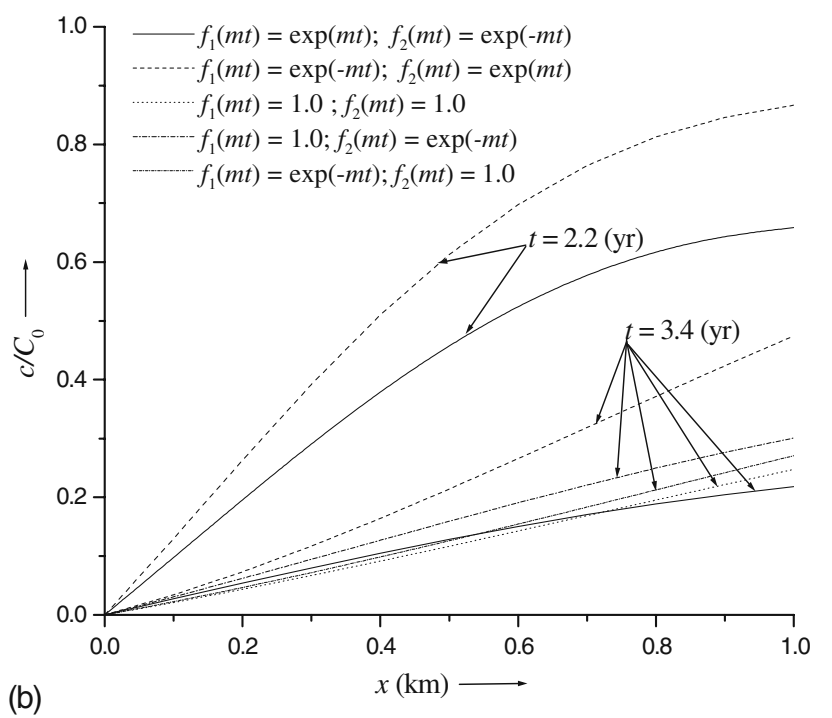

Figure 2. Comparison of solute transport in five cases of unsteadiness of dispersion and the flow through inhomogeneous medium (a) in the presence of the uniform pulse-type point source and (b) in the absence of the uniform pulse-type point source.

One time-dependent form $f(m t)=\exp (m t)$ tends to infinity as $t \rightarrow \infty$; hence, it may be argued that ultimately the solute transport or velocity of the flow field increases enormously. But this situation will never occur because, as observed from the figures, after a certain time period, an equilibrium stage is reached and the source becomes ineffective with regard to it polluting the domain. Once the source is removed, the rehabilitation process is almost completed in a finite time period. The unsteadiness and inhomogeneity in different media will vary. In air the two may be on the higher side, while in soil and in aquifer they will be on the lower side. The present study may be useful to account for the dispersion through any medium 
by appropriate choice of values of the respective parameters. In aquifer, the velocity will satisfy the Darcy law and its value may be chosen between $2 \mathrm{~m} /$ day and $2 \mathrm{~m} /$ year (Todd 1980). The nomenclature of the dependent variable in different disciplines stated at the outset of section 1 may be done accordingly, e.g., in wound healing the variable $c$ will represent cell concentration or the growth factor (chemical) concentration. Additionally, such study will be useful particularly in:

- validating numerical solutions of more real dispersion problem,

- in assessing the rehabilitation process of a polluted domain once the source of pollution is eliminated forever, and

- in assessing the unsteady nature of dispersion of the pollutants and advection, and heterogeneity of the medium.

Further, as with the chosen values of $u_{0}, D_{0}$, inhomogeneity parameter, $a$, and unsteadiness parameter, $m$, the variations in velocity and dispersion remain of small order, and so the Fickian law of conservation of solute mass is not disobeyed.

\section{Conclusion}

The increasing demand for analytical solutions of $\mathrm{ADE}$, with variable coefficients in more realistic and general forms, has motivated us to obtain one such solution by using a simpler LITT. Particular solutions may be obtained from it for less real and ideal cases. With the help of new independent variables introduced through separate transformations at the different stages, the variable coefficients were reduced to constant coefficients. The source of pollution is considered to be stationary uniform point source of pulse type. The independent functions for the unsteadiness of solute dispersion, flow and the inhomogeneity of the medium are the key features of the present study. More time-dependent expressions for $f_{1}\left(m_{1} t\right)$ and $f_{2}\left(m_{2} t\right)$ may be chosen for which an explicit relationship, such as the one as in equation (26), may be derived. In future work, a moving point source (Crank 1987) may be considered. Additional effects on the solute transport due to adsorption, first-order decay and zerothorder production may also be modelled and studied (Toride et al 1993). This work may be extended to two- and three-dimensions.

\section{Acknowledgements}

The authors express their gratitude to the learned reviewers for their extremely valuable comments and suggestions that improved the present work to a great extent.

\section{References}

Brenner H 1962 The diffusion model of longitudinal mixing in beds of finite length - numerical values; Chem. Eng. Sci. 17 229-243.

Cassol M, Wortmann S and Rizza U 2009 Analytic Modeling of two-dimensional transient atmospheric pollutant dispersion by double GITT and Laplace Transform techniques; Environ. Mod. Software 24 144-151.

Chen J S, Ni CF, Liang C P and Chiang C C 2008 Analytical power series solution for contaminant transport with hyperbolic asymptotic distance-dependent dispersivity; J. Hydrol. 362 142-149.

Costa C P, Vilhena M T, Moreira D M and Tirabassi T 2006 Semi-analytical solution of the steady three-dimensional advection-diffusion equation in the planetary boundary layer; Atmos. Environ. 40(29) 5659-5669.

Cotta R M and Mikhailov M D 1997 Heat conduction: Lumped analysis, integral transforms, symbolic computation, John Wiley, pp. 352.

Crank J 1975 The mathematics of diffusion, Oxford University Press.

Crank J 1987 Free and moving boundary problems, Oxford University Press.

Demuth C 1978 A contribution to the analytical steady solution of the diffusion equation for line sources; Atmos. Environ. 12 1255-1258.

Frippiat C C and Holeyman A E 2008 A comparative review of upscaling methods for solute transport in heterogeneous porous media; J. Hydrol. 362 150-176.

Golz W J 2003 Solute transport in a porous medium: A mass-conserving solution for the convection-dispersion equation in a finite domain; Ph.D. thesis, Louisiana State University.

Guerrero J S P, Pimentel L C G, Skaggs T H and van Genuchten M Th 2009 Analytical solution of the advection-diffusion transport equation using a change-ofvariable and integral transform technique; Int. J. Heat Mass Transfer 52 3297-3304.

Jaiswal D K, Kumar A, Kumar N and Yadav RR 2009 Analytical solutions for temporally and spatially dependent solute dispersion of pulse type input concentration in onedimensional semi-infinite media; J. Hydro-environ. Res. 2 254-263.

Kumar A, Jaiswal D K and Kumar N 2009 Analytical solutions of one-dimensional advection-diffusion equation with variable coefficients in a finite domain; J. Earth Syst. Sci. 118 539-549.

Kumar A, Jaiswal D K and Kumar N 2010 Analytical solutions to one-dimensional advection-diffusion equation with variable coefficients in semi-infinite media; J. Hydrol. $380330-337$.

Lindstrom F T and Boersma L 1989 Analytical solutions for convective-dispersive transport in confined aquifers with different initial and boundary conditions; Water Resour. Res. 25 241-256.

Marino M A 1974 Distribution of contaminants in porous media flow; Water Resour. Res. 10 1013-1018.

Matheron G and de Marsily G 1980 Is transport in porous media always diffusive? A counterexample; Water Resour. Res. 16 901-917.

Mikhailov M D and Ozisik M N 1984 Unified analysis and solutions of heat and mass diffusion, John Wiley \& Sons.

Moreira D M, Vilhena M T, Buske D and Tirabassi T 2009 The state-of-art of the GILTT method to simulate pollutant dispersion in the atmosphere; Atmos. Res. 92 1-17.

Neelz S 2006 Limitations of an analytical solution for advection-diffusion with spatially variable coefficients; Commun. Numer. Methods Eng. 22 387-396. 
Ogata A 1970 Theory of dispersion in granular media; US Geol. Sur. Prof. Paper 411-I, 34.

Rounds W 1955 Solution of the two-dimensional diffusion equation; Trans. Am. Geophys. Union 36 395-405.

Rumer R R 1962 Longitudinal dispersion in steady and unsteady flow; J. Hydraul. Div. 88 147-172.

Scheidegger A E 1957 The Physics of flow through porous media, University of Toronto Press.

Scriven R A and Fisher B A 1975 The long range transport of airborne material and its removal by deposition and washout-II. The effect of turbulent diffusion; Atmos. Environ. 9 59-69.

Selim H M and Mansell R S 1976 Analytical solution of the equation of reactive solutes through soils; Water Resour. Res. 12 528-532.

Shevelev A G 1969 Application of the Laplace transform to the solution of linear differential equations with variable coefficients; Ukranian Math. J. 21(5) 538-547.

Shukla J B, Mishra A K and Chandra P 2008 Mathematical modeling and analysis of the depletion of dissolved oxygen in eutrophied water bodies affected by organic pollutants; Nonlinear Anal.: Real World Appl. 9 1851-1865.
Smedt F De 2007 Analytical solution and analysis of solute transport in rivers affected by diffusive transfer in the hyporheic zone; J. Hydrol. 339 29-38.

Smith F B 1957 The diffusion of smoke from a continuous elevated point source into a turbulent atmosphere; $J$. Fluid Mech. 2 49-76.

Todd D K 1980 Groundwater Hydrology; 2nd edn, John Wiley \& Sons.

Toride N, Leij F J and van Genuchten M Th 1993 A comprehensive set of analytical solutions for nonequilibrium solute transport with first order decay and zero order production; Water Resour. Res. 29 2167-2182.

van Genuchten M Th and Alves W J 1982 Analytical solutions of the one-dimensional convective-dispersive solute transport equation; US Dept. Agriculture Tech. Bull. No. 1661151.

Wortmann S, Vilhena M T, Moreire D M and Buske D 2005 A new analytical approach to simulate the pollutant dispersion in the PBL; Atmos. Environ. 39 2171-2178.

Zoppou C and Knight J H 1999 Analytical solution of spatially variable coefficients advection-diffusion equation in up to three dimensions; Appl. Math. Mod. 23 667-685. 